\title{
Intelligent Healthcare Monitoring using Radio Frequency Identification Detection
}

\author{
Abdul Razaque \\ Department of Electrical \\ \& Computer Science \\ Cleveland State \\ University
}

\author{
Fathi Amsaad \\ Department of Electrical \\ \& Computer Science \\ University of Toledo, \\ Toledo, $\mathrm{OH}, \mathrm{USA}$
}

\author{
Musbah Abdulgader \\ Department of Electrical \\ \& Computer Science \\ University of Toledo, \\ Toledo, $\mathrm{OH}, \mathrm{USA}$
}

\author{
Bhanuteja Deekonda \\ Department of Electrical \\ \& Computer Engineering \\ Cleveland State \\ University
}

\section{Chandra Sekhara Chinta Department of Electrical \& \\ Computer Engineering \\ Cleveland State University}

\author{
Soumya Susan lype \\ Department of Electrical \& \\ Computer Engineering \\ Cleveland State University
}

\author{
Naveen Tankala \\ Department of Electrical \& \\ Computer Engineering \\ Cleveland State University
}

\begin{abstract}
The use of Radio Frequency Identification technology enhances efficiency and security of many real-time systems. The features of RFID makes it adaptable in many fields such as transportation, security, agriculture, healthcare focusing mainly on tracking and storage of information. The addition of RFID system to the healthcare sector would improve overall efficiency of system with minimum cost of maintenance for the long run. The medical history of a patient could be stored electronically eliminating the need of storing paper-based patient files. By automating the healthcare sector, records are readily available to doctors which are very helpful in taking decisions and providing immediate care to the patients. This paper proposes Intelligent Healthcare Monitoring using RFID technology and secure ZigBee networking.
\end{abstract}

This aims to validate data accuracy and confidentiality. The proposed system is supported using the algorithms, Record Duplication Checking (RDC) and Automatic Patient Record Reading Process (APRRP). Our proposed system is also tested using Ultra High Frequency RFID and mesh network of ZigBee devices. Based on the experimental results, we proved that the proposed Intelligent Health Care System could be implemented in real-time healthcare facilities where the patient medical data is stored automatically without much human intervention.

\section{General Terms}

Design, Development, Testing

\section{Keywords}

RFID; ZigBee; Intelligence Health Monitoring; Medical records; Tag

\section{INTRODUCTION}

Proper healthcare monitoring is one of the major issues faced by healthcare sector. Many people face medical issues due to improper diagnosis as a result of flaws in management of patient's medical records. There is a need to ease the life of patients by automating the healthcare system whereby medical details of patients are stored with accuracy and security. The medical details of a person can be stored in private by providing read/write access of records to only required personnel. Also, the medical condition of patients moving between different healthcare facilities if tracked would be helpful in taking immediate decisions and provide proper care to patients. The scope of this system could be extended where the stay of patient at hospital is reduced by secure communication between patient and hospital facilities.

The introduction of Radio-frequency Identification (RFID) into Healthcare [1] is an economic solution for a reliable patient healthcare monitoring system. In RFID systems, there are RFID tags that stores data which communicates through radio signals produced by RFID readers [2]. The operation of RFID do not require line-of-sight and has capability to read or write making technology more reliable [3]. Since RFID tags provides current status information or data about patients electronically, there is a decline in errors while making decisions and also ensures the safety of a patient during a medical emergency.

A typical RFID system comprises of a RFID tag, RFID reader and a middleware interface that sends data to required location. The RFID tag has a chip with memory and an antenna to accept radio signal from RFID reader. The tags are either passive or active. The passive ones are preferred in Healthcare monitoring due to their flexibility and greater life with no power requirement. RFID reader has an antenna that generates field for communication with RFID tag. An efficient communication between RFID components is possible only if they are properly integrated and are made compatible with each other [4].Usually, RFID systems operate at frequency ranges of Low frequency (125 to $148 \mathrm{KHz}$ ), High Frequency (13.56 MHz), Ultra High Frequency (UHF$915 \mathrm{MHz}$ ) or Microwave (MW-2.45 GHz).Since long distant communication and speed of operation is an important factor in design of RFID components, UHF or MW is preferred over other frequency ranges. Also, size of antennas is small for UHF or MW RFID systems whereby making system more compact. The power-transmission efficiency and quality of data transmitted depends on choice of network.

The paper discusses about two networks that provides efficient communication between patients and medical facilities. A standard RFID communication at $915 \mathrm{MHz}$ is responsible for sending medical details from RFID tag provided for a person to node. The data from node is sent to 
required medical facility through ZigBee network which is a simple, cost-effective technology requiring less power. The data is transmitted over long distances by a mesh network of ZigBee devices with proper energy efficiency and secure networking but at a small data transfer rate [5]. Thus, various advantages and issues in implementing RFID into healthcare monitoring is discussed in the paper.

The paper discusses the implementation of RFID technology into Healthcare Monitoring. Section 2 is an overview of problem identification in RFID communication and its significance. Section 3 gives an overview of existing approaches in the literature survey. Section 4 presents proposed intelligent health care system. Section 5 shows integration process of RFID AND ZigBee. Section 6 presents an experimental results and discussion. Section 7concludes the paper and references are given in section 8 .

\section{PROBLEM IDENTIFICATION AND SIGNIFICANCE}

An analysis of RFID field and study of power transmission is also done, which is important for stability of system and accuracy of the data being transmitted. Since passive tag is utilized in system that takes power from the reader making it mobile, a low power analog signal conversion circuit needs to be designed.

Noise resistivity is one of the issues in RFID communication as environment or movement of a person induces noise. The electronic devices are capable of producing transients that might interfere with RF communication reducing accuracy of the whole system.

Other issues are failure to read tags and duplication of data [6]. Failure to read tags might be due to improper orientation of tag or absorption or reflection of radio waves. If there is duplication of data, the overall life of the network is reduced as there are chances of irrelevant data transfers that uses the energy of nodes in network.

It is important that above identified problems be resolved to improve the efficiency of system. In healthcare monitoring, passive tags are preferred considering cost and mobility of system. The study of power consumed by RFID components is important to design additional circuitry with less power requirements.

If active tags were used, there is always a need of power supply to charge tags which might bother patients.

Accuracy and security of patient medical information recorded and transmitted is very important [7]. It is based on these data that evaluation or diagnosis of a patient is done remotely. Thus, it should be ensured that even the latest medical record is available at a medical facility for proper monitoring and guidance. The components of RF system should be compatible to each other and proper networking will improve the overall performance of system. The addition of ZigBee network with low power radio waves guarantees secure networking in a medical facility [8].

\section{RELATED WORK}

We introduce some interesting and salient features into the existing approaches. The errors happening in the healthcare sector has resulted in death of people which could have been avoided if proper technologies to minimize errors were incorporated into the healthcare sector [9].It is time consuming to manually add all the patient information and there are chances of errors.

Barcode technology in healthcare helped in tracking of patients during their stay in hospitals. The tracking of medical records, drugs and specimens of patients was possible [10] using barcode. This enhanced the safety and care given to patients by providing correct treatment as a result of less errors. The bar codes are cheap, simple and easy to use. The main problem with bar code scanning is that they require line of sight thus demanding a need of human involvement for scanning [11].

The RFID implementation in the healthcare sector is helpful in many ways such as patient tracking, pharmaceutical inventory and tracking of medical instruments [12]. In health care, initially RFID was confined to objects for their tracking. RFID does not require the line-of-sight scanning reducing the human assistance and hence system is automated. The cost of implementation of a technology is a major concern and RFID technology is a solution for long term systems.

Since RFID could not be applied in large-scale areas, the addition of Bluetooth or IEEE 802.11 solved this problem which has good data transfer rates [13]. Bluetooth system is efficient in small area networking as almost 7 nodes would be activated at a time. But, hospital data networking requires data networking on a large scale. ZigBee networking along with RFID readers in ad hoc pattern would be helpful in extending the communication range of the RFID tags.

Several factors need to be considered before implementing RFID into the healthcare sector. As providing the best possible care to patients is the ultimate motive behind automation of the healthcare sector, a deep research of various issues is very important.

\section{PROPOSED INTELLIGENT HEALTHCARE SYSTEM}

We focused on the implementation of RFID in healthcare monitoring as it will automate the medical sector whereby medical records of patients are recorded and stored without much human intervention. An electronic patient record eliminates the need to store paper based patient records and also helps doctors to make proper diagnosis and care to patients. Passive RFID tags with capability to read and write medical records could be assigned to patients with various IDs.

Radio waves in the frequency range of $800-950 \mathrm{MHz}$ (Ultra High Frequency) is preferred because of small antenna size, longer ranger and data transfer rate. The design of RFID components in UHF also avoids interferences of other possible wireless networks in the vicinity. As passive tags use energy of the reader for communication, adding a low power circuitry to the tags for data acquisition will reduce the actual power requirement of tag. There will be a leakage of data signal from tags that reduces sensitivity because of noise. The transmitter and receiver must be isolated using circuits. The cost of tags increase with storage size and must be available in larger scale and at an economical rate. Thus, periodical 
removal of data from tags and storage in computer would allow hospitals to use RFID tags more effectively.

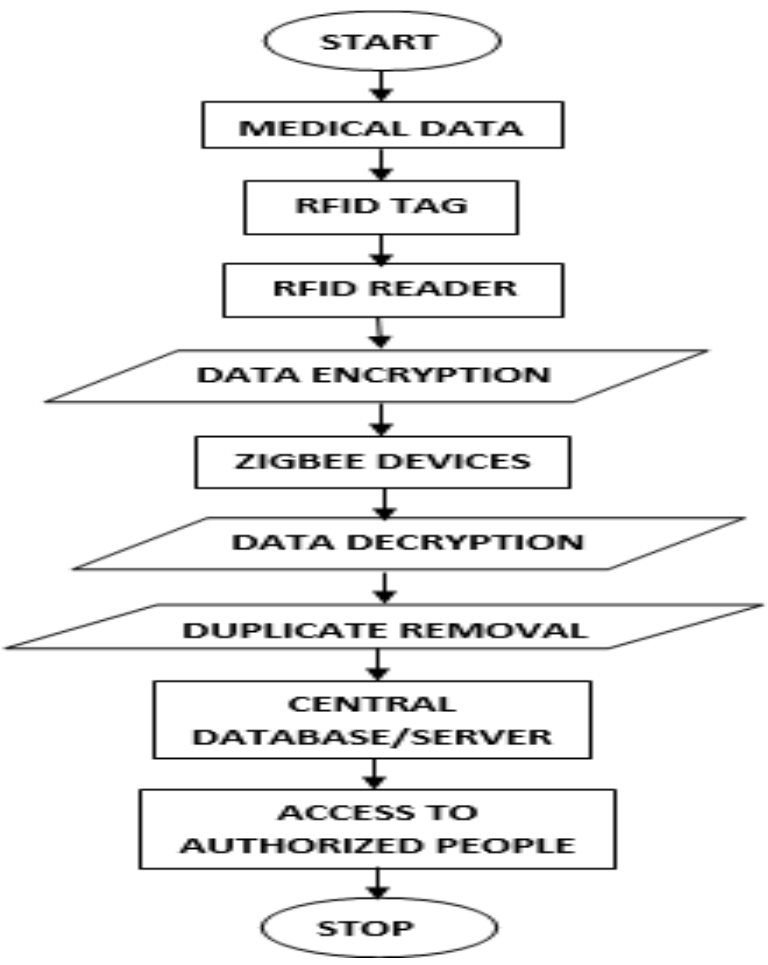

Figure 1: Proposed flowchart of RFID System

In order to ensure tag reading, the reader is made mobile by adopting tree topology where different readers are connected through a wireless network. The networking of data using ZigBee devices is efficient as it has less power dissipation compared to other latest wireless communication technologies like Wireless LAN or Bluetooth which are costlier and has less range. The data transfer rate is less in ZigBee devices but shouldn't be of much concern in healthcare monitoring.

As security and privacy of patient data is important, proper encryption and decryption of data is necessary. The data stored in tag must be encrypted and should be decrypted only at required server. Thus data is made accessible only to authorized personnel ensuring security. Smoothing of RFID data removing noise and elimination of duplicate data will improve the overall performance of system. The life of the network is affected with duplicated data transfers as it utilizes the energy of nodes in network. By adding window approach, noise and data duplication could be eliminated.

The complete intelligence health care system process is depicted in the Fig 1. Medical data is stored in the RFID tag. The tag is read by the reader. If any noise or duplication of data is found, the reader will read the tag again. Or else, the data is encrypted. This encrypted data is sent through wireless networking device ZigBee. The encrypted data is then decrypted and stored in the database/servers. Only authorized medical representatives or Hospital management can access the data.

\section{INTEGRATION PROCESS OF RFID AND ZIGBEE}

Our methodology helps in selecting several hardware and software equipment which are low of cost as well as efficient meeting our objective to the best. The Integration of Ultra
High Frequency driven RFID system with a mesh network of ZigBee makes the data transfer more reliable and secure.

\subsection{Ultra-High Frequency driven RFID System}

The hardware components include RFID tags and RFID readers. Passive RFID tags in frequency range of 800-950 $\mathrm{MHz}$ are selected for hospital purposes. As the size of the antenna is dependent on the wavelength, a high frequency is preferred for producing compact tags. Also, the data transfer rate is more for higher frequencies.

\begin{tabular}{|c|}
\hline $\begin{array}{l}\text { Algorithm 1: Automatic Patient's Record } \\
\text { Reading Protocol(APRRP) }\end{array}$ \\
\hline 1. Initialization (Rtag : RFID tag \\
\hline Pn : Patient name \\
\hline Pr : Patient reports \\
\hline Prep : Previous report \\
\hline Nrep : New report ） \\
\hline 2.Input $\quad(\mathrm{Hr}: \mathrm{RFID}$ tag $\quad)$ \\
\hline 3.Output ( Pr: Patient reports ) \\
\hline 4.Set Rtag \\
\hline 5.Calculate Rtag $=\operatorname{Pn}+\operatorname{Pr}$ \\
\hline 6.If ( $\mathrm{Nr}:$ New report to be updated ) \\
\hline 7.Prep $=\mathrm{Pr}+\mathrm{Nr}$ \\
\hline 8.Else \\
\hline 9.Prep $=\operatorname{Pr}$ \\
\hline 10.End if \\
\hline 11.End else if \\
\hline
\end{tabular}

These tags will have a unique reference code and are identity of patient which is the primary field in database. For the data storage, we intend to use EEPROM (Electrically Erasable Programmable Read Only Memory) with a data storage of 640bit and CMOS fabrication. Since passive tags require power from the reader for communication, a rectifier circuit with regulators provides the required power for the operation of ICs in the tag. The circuitry process the energy, utilizes it and retransmits the recorded data in tag back to the reader. RFID readers will read data from RFID tags. It can send power as well as data. Different readers might be collecting the same reading of a tag at the same time. All these data would be eventually going to a central server and there is a need to filter these data through some programming techniques. A computer/server will store data from tags through proper networking. The tag numbers are the primary field in the data base and medical data is stored based on these numbers.

The APRRP algorithm is the algorithm which checks whether a new patient record should be added to the existing electronic patient file. First, RFID tag, patient name, patient reports, previous report, and new report are initialized as the variables. Input is the RFID tag and output is the patient reports. When the tag is read, the patient name and previous reports are 
available. If any new reports $(\mathrm{Nr})$ are to be updated, previous reports and new reports are added to the patient reports. If not, previous reports will remain same and the statements following if statement is ended.

\subsection{Integration of ZigBee Framework}

As hospitals require data transmission on a large scale, we propose to transfer the stored data in medical facilities using ZigBee devices. A mesh network of ZigBee devices are preferred over other wireless networks due to low cost and less power dissipation [15]. The range of these devices will increase if data is made to hop node to node. The system is made more reliable by adding the "Self-healing" capability where alternate path is followed when a connection is lost or when a node fails. A simple ZigBee Mesh network comprises of three things.

- An end device which is a computer or mobile.

- A router which routes the traffic between nodes

- A coordinator which is a special router responsible for forming a network. The Coordinator authenticates new nodes and distribute network keys to the nodes.

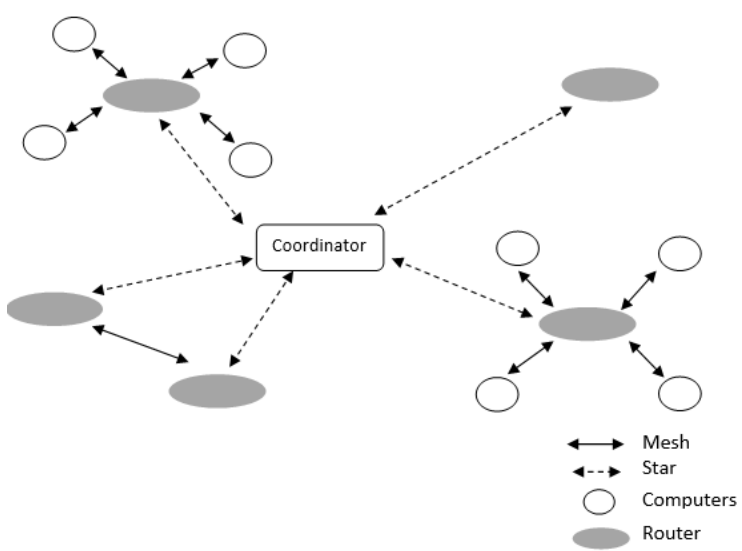

Figure 2. Framework of ZigBee Networking

\subsection{Data Accuracy}

The filtering of RFID data by adding algorithms will minimize errors in data recorded. By providing multiple reading cycles to the data from the readers, the duplicate data could be eliminated. We stored the RFID information in the format (reader_id, tag_id, patient_data, time).

The RRD algorithm removes the duplicate records due to multiple readers for a single RFID tag. The initialization of the parameters are done in line1.The updated records of the patient after duplicate removal is the output. The repetition of a patient record is compared using an update Checker, recorded RFID tag number and Time Stamp of the recorded data. Only first record is retained and rest of the duplicated records are deleted.

\begin{tabular}{c}
\hline Algorithm 2: Removing Record Duplication(RRD) \\
\hline 1. Initialization (Rtag : RFID tag \\
Rid: Reader id \\
Pn : Patient name \\
Pr : Patient record
\end{tabular}

\author{
Ts: Time Stamp \\ TotPr: Total Patient Records \\ $\Delta$ insert: Insert checker \\ ResRtag : Result Rtag \\ ResTs: Result TimeStamp )
}

2. Input ( $\mathrm{Hr}: \mathrm{Rid}, \mathrm{Ts}$,)

3. Output (Pr: Rtag, Rid, Pn, Pr, Ts)

4. for $(\mathrm{i}=0 ; \mathrm{i}<\mathrm{TotPr} ; \mathrm{i}++)$

5. Set $\Delta$ insert $=1$

6. for $(j=0 ; j<\operatorname{TotPr} ; j++)$

5.if $((\operatorname{Rtag}==$ ResRtag $)$ then

6. if(Ts $==$ ResTs) then

Set $\Delta$ insert $=0$

7. end if

8.Repeat step 6

9. if $(\Delta$ insert $==1)$

Set Pr=( Rtag, Rid, Pn, Pr, Ts)

10.end if

\section{EXPERIMENTAL RESULT AND DISCUSSION}

The Automatic Patient's Record Reading protocol (APRRP) is implemented using java platform. The Removing Record Duplication (RRD) algorithm is implemented in the server side. C\# code and the .NET platform verified the algorithm and duplicate patient data is successfully removed. Thus, storage capability of the system is enhanced and the overall performance of the system is improved. The complete implementation parameters are given in Table 1.

Table 1: Parameters used in simulation

\begin{tabular}{|l|l|}
\hline \multicolumn{1}{|c|}{ Parameters } & \multicolumn{1}{c|}{ Description } \\
\hline Rtag & RFID tag given to the patient \\
\hline Rid & RFID reader \\
\hline Pn & Patient name stored in data. \\
\hline Pr & Patient records \\
\hline Ts & Time stamp of recorded data \\
\hline TotPr & Total reports of the patient \\
\hline$\Delta$ insert & $\begin{array}{l}\text { Checks whether record need } \\
\text { to be inserted }\end{array}$ \\
\hline Nrep & New reports of the patient \\
\hline Prep & $\begin{array}{l}\text { Previous reports of the } \\
\text { patient }\end{array}$ \\
\hline
\end{tabular}

We have used java platform to execute the algorithm. The results are shown in above figures. In Figure 3 and Figure 4 Patient reports are being entered into the database. Thereby another patient reports are also added to the database with their name, DOB, ID and their diseases. In Figure 5 we can see how to get all the reports present in the database and also 
how to get a particular patients' reports by entering their assigned id number. We also ensured the patient records are not repeated during storage. The patient records for a particular timestamp would cause a duplication of data.

These duplicate data is filtered at the server side before sending it to the client side. Figure5 shows the patient records where more than one reader reads the same patient record at the same timestamp. Figure 6 shows the patient records after duplicate removal using the RRD algorithm implementation. As a result, the memory and the speed of the system are improved.

\section{CONCLUSION}

Proper Healthcare Monitoring is an issue in the healthcare sector, especially with the electronic storage and accuracy of medical records of a person. The Intelligent Healthcare Monitoring uses Radio Frequency Identification Detection technique where medical details of a patient are stored using RFID tags. The data from the RFID tags is read by RFID readers and is distributed on a large scale using ZigBee network which has less power dissipation compared to other technologies. The Automatic Patient's Record Reading Protocol (APRRP) algorithm stores new. The accuracy of patient records is ensured using Removing Record Duplication (RRD) algorithm.

\section{REFERENCES}

[1] Lee, Shuenn-Yuh, Liang-Hung Wang, and Qiang Fang. "A low-power RFID integrated circuits for intelligent healthcare systems." Information Technology in Biomedicine, IEEE Transactions on 14, no. 6 (2010): 1387-1396.

[2] Ayoade, John, and Judith Symonds. "An Evaluation of the RFID Security Benefits of the APF System: Hospital Patient Data Protection." International Journal of Advanced Pervasive and Ubiquitous Computing 1, no. 1 (2009): 44-59.

[3] Reiner, J., and M. Sullivan. "RFID in healthcare: a panacea for the regulations and issues affecting the industry." Healthcare Purchasing News (2005).

[4] Ahsan, Kamran. RFID components, applications and system integration with healthcare perspective. INTECH Open Access Publisher, 2011.
[5] Somani, Nisha Ashok, and Yask Patel. "Zigbee: A Low Power Wireless Technology For Industrial Applications." International Journal of Control Theory and Computer Modelling (IJCTCM) Vol 2 (2012).

[6] Bai, Yijian, Fusheng Wang, and Peiya Liu. "Efficiently Filtering RFID Data Streams." In CleanDB. 2006.

[7] Juels, Ari. "RFID security and privacy: A research survey." Selected Areas in Communications, IEEE Journal on 24, no. 2 (2006): 381-394.

[8] Ball, Edward, David W. Chadwick, and Darren Mundy. "Patient privacy in electronic prescription transfer." Security \& Privacy, IEEE 1, no. 2 (2003): 77-80.

[9] McCathie, Luke. "The advantages and disadvantages of barcodes and radio frequency identification in supply chain management." Faculty of Informatics-Theses (2004): 9 .

[10] Coustasse, Alberto, Shane Tomblin, and Chelsea Slack. "Impact of radio-frequency identification (RFID) technologies on the hospital supply chain: a literature review." Perspectives in Health Information Management 10, no. Fall (2013).

[11] Bridgelall, Raj. "Bluetooth/802.11 protocol adaptation for RFID tags." In Proceedings of the 4th European Wireless Conference. 2002.

[12] Cao, Huasong, Victor Leung, Cupid Chow, and Henry Chan. "Enabling technologies for wireless body area networks: A survey and outlook." Communications Magazine, IEEE 47, no. 12 (2009): 84-93.

[13] Dubin, Cindy H. "Bar-code scanning at four health care facilities in the US." Pharmacy and Therapeutics 35, no. 4 (2010): 212.

[14] Korkmaz, Ilker, Coskun Atay, and George Kyparisis. "A mobile patient monitoring system using RFID." Latest Trends on Computers 2 (2010): 726-732.

[15] Razaque, Abdul, and Khaled Elleithy. "Automatic energy saving (AES) modelto boost ubiquitous wireless sensor networks (WSNs)." arXiv preprint arXiv:1309.4504 (2013). 
Communications on Applied Electronics (CAE) - ISSN : 2394-4714

Foundation of Computer Science FCS, New York, USA

Volume 4-No.5, February 2016 - www.caeaccess.org

\section{APPENDIX}

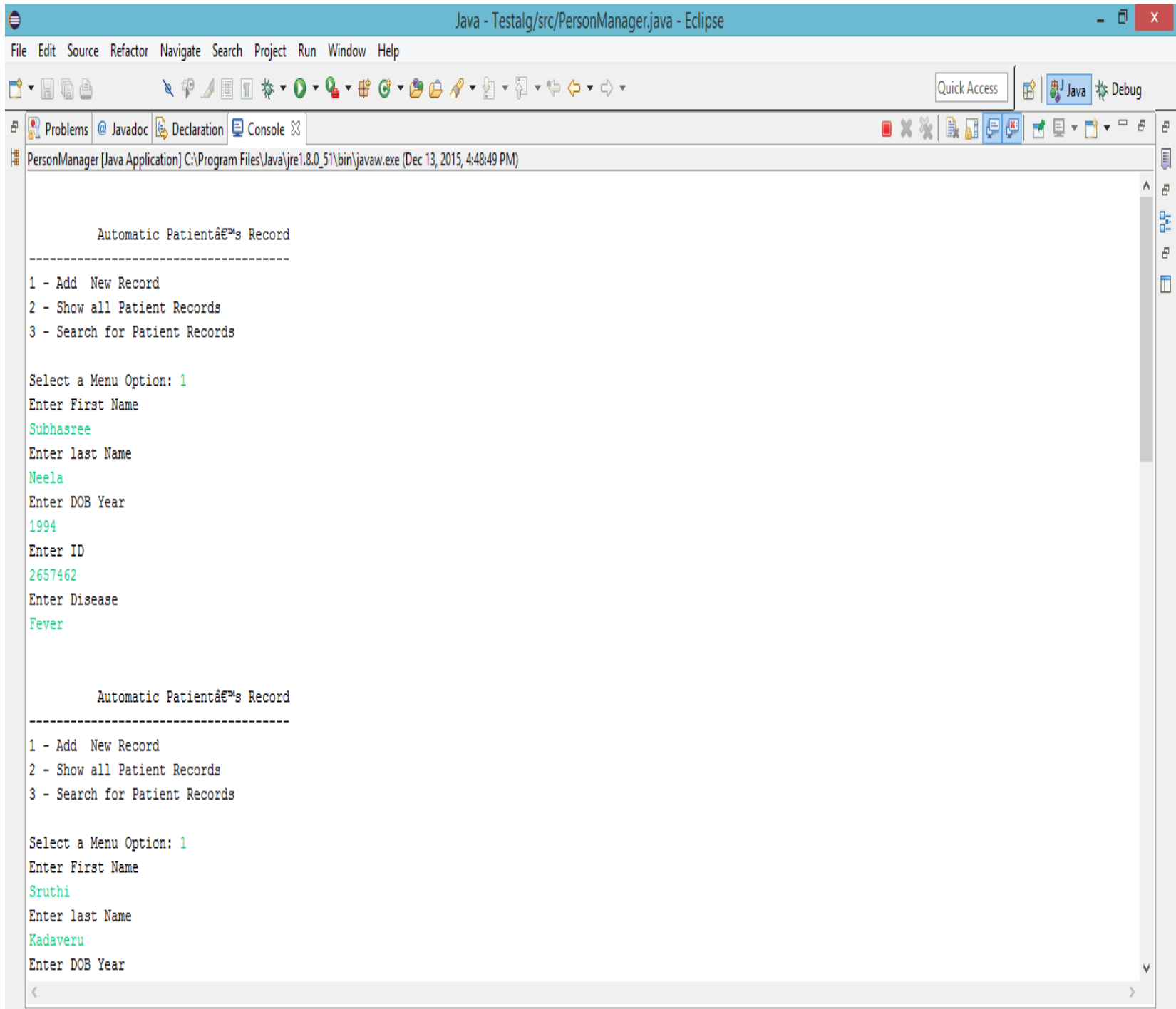

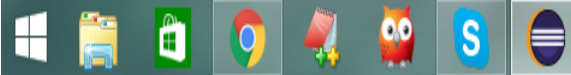

Figure 3: Entering patient records 


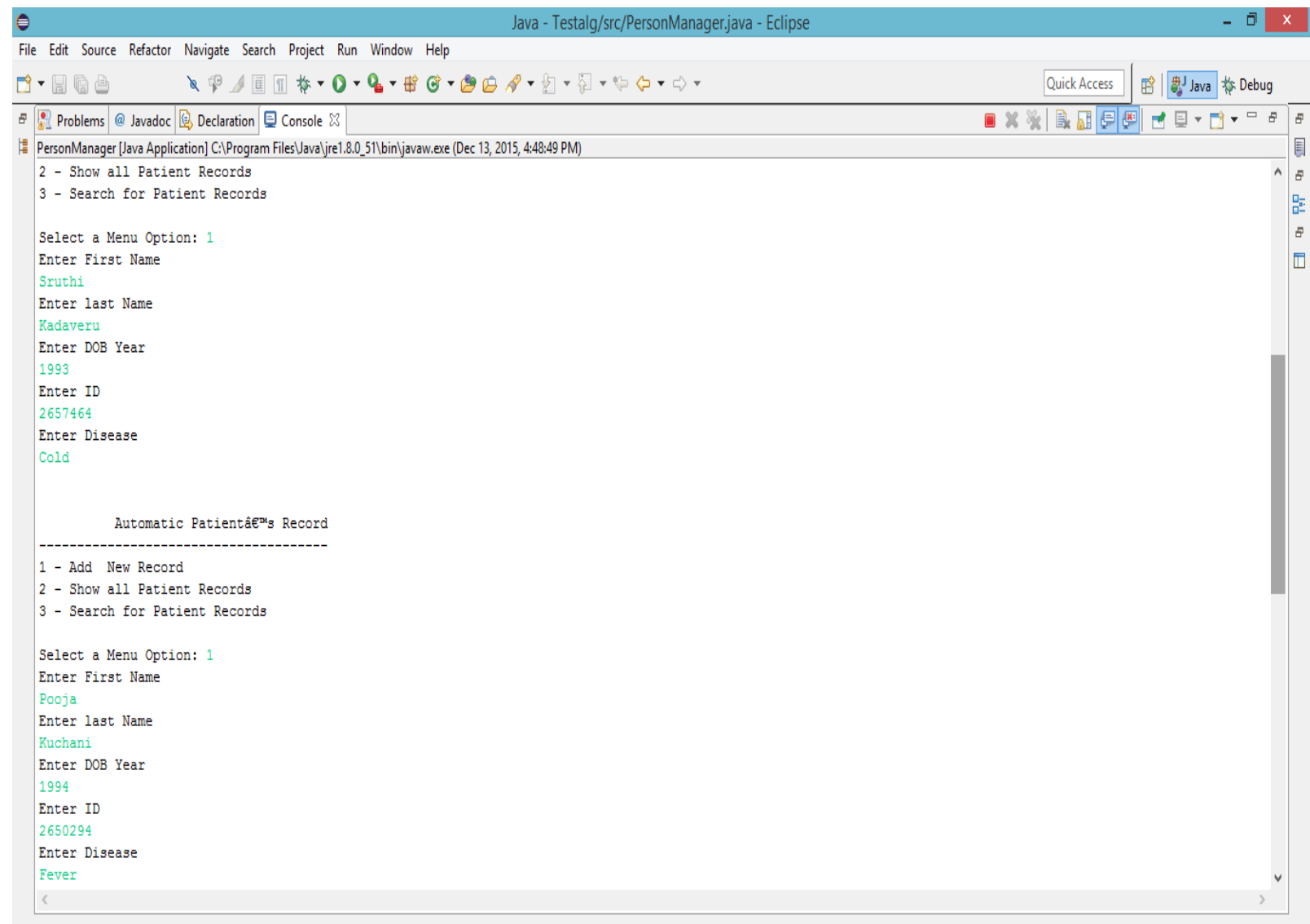

\section{明的}

Figure 4: Adding other patient's reports

\begin{tabular}{|l|l|l|l|l|}
\hline Rtag & Patient_Name & Patient_Record & Time_Stamp & \multicolumn{1}{l|}{ R_id } \\
\hline 125 & Soumya & ECG & 1 & 1250 \\
\hline 125 & Soumya & ECG & 2 & 1251 \\
\hline 125 & Soumya & ECG & 2 & 1252 \\
\hline 120 Sundar & ECG & 1 & 1250 \\
\hline 122 & Bhanu & ECG & 1 & 1251 \\
\hline 120 & Sundar & ECG & 2 & 1252 \\
\hline 120 & Sundar & ECG & 2 & 1253 \\
\hline 125 & Soumya & ECG & 1 & 1254 \\
\hline
\end{tabular}

Figure 5: File with duplicate records 


\begin{tabular}{|l|l|l|l|l|}
\hline Rtag & Patient_Name & Patient_Record & Time_Stamp & R_id \\
\hline 125 Soumya & ECG & 1 & 1250 \\
\hline 125 Soumya & ECG & 2 & 1251 \\
\hline 120 Sundar & ECG & 1 & 1250 \\
\hline 122 Bhanu & ECG & 1 & 1251 \\
\hline 120 Sundar & ECG & 2 & 1252 \\
\hline
\end{tabular}

Figure 6: File after duplicate removal

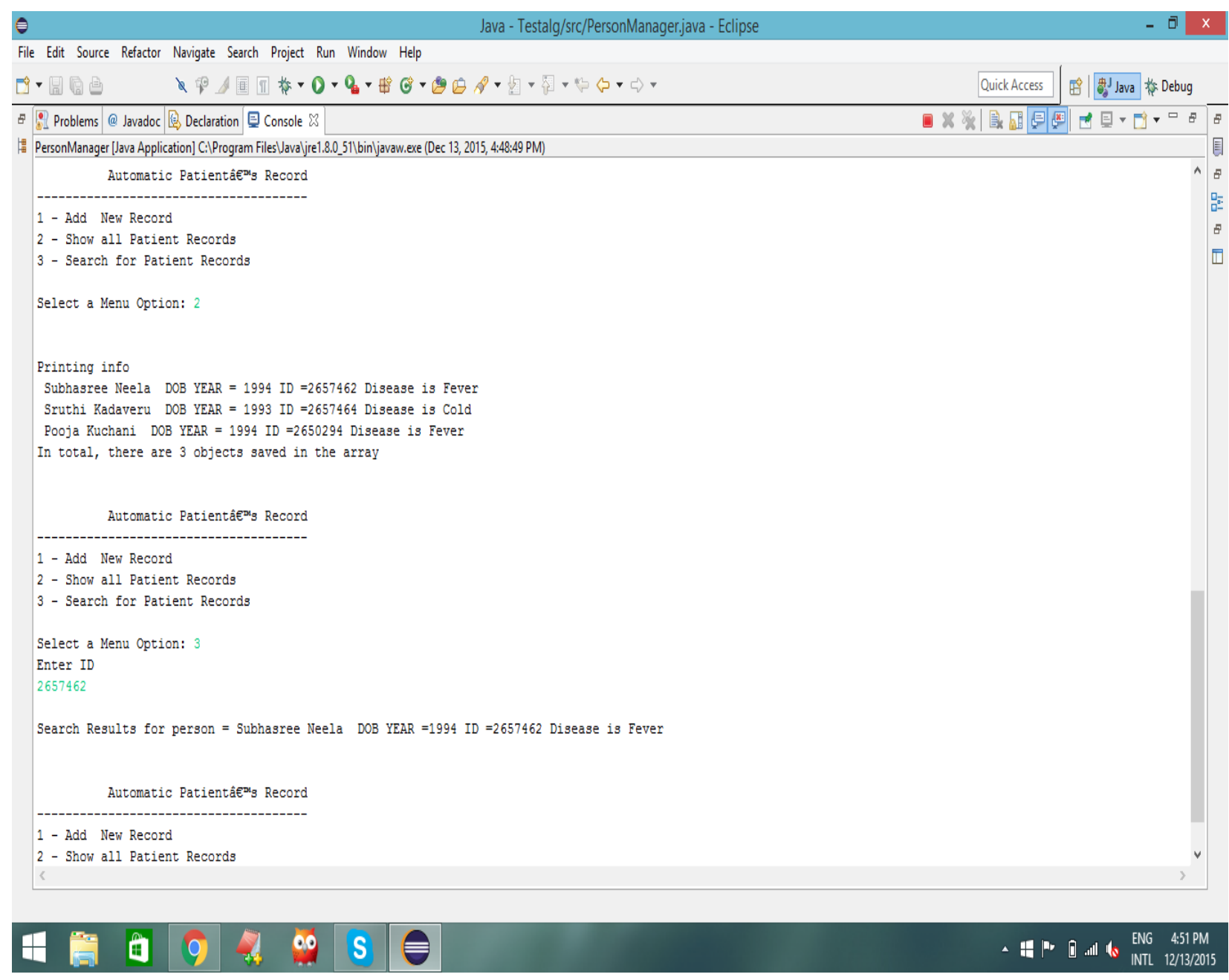

Figure 7: Displaying all patient records and getting patient reports by patient id 